\title{
Discrete Time Optimal Harvesting of Fish Populations with Age Structure
}

\author{
Wandi Ding ${ }^{1, *}$, Suzanne Lenhart ${ }^{2}$, Horst Behncke ${ }^{3}$
}

\begin{abstract}
We consider an optimal fishery harvesting problem using an age-structured population model with nonlinear recruitment. The motivating example is Atlantic Cod. The goal is to maximize the profit (total gain) of fishing. We seek to find the optimal harvesting strategy for each age class, but also to find the optimal net size. Using the extension of Pontryagin's Maximum Principle to discrete systems, we are able to derive the necessary conditions and the characterizations for the optimal harvesting strategies. Numerical simulations for both Beverton-Holt and Ricker recruitment functions are provided.
\end{abstract}

Keywords: optimal harvesting, discrete model, age structure

\section{Introduction}

Fish is a valuable source of protein, and many people have lived to a large extent on fish and other seafood. Overfishing has driven many stocks of fish to near extinction $[9,10]$. This applies in particular to the Mediterranean Sea, the Baltic Sea, and the North Atlantic Ocean. The collapse of the Newfoundland or Baltic sea cod fisheries should be taken as a severe warning that the fishery industry needs more careful controls [13]. With appropriate stock assessment data, mathematical models can be used to derive possible management strategies, which may aid the supervision of this industry.

The fundamental work on quantitative fisheries management was done by Beverton and Holt, Ricker, Schaefer and Clark [2, 4, 25, 26, 27, 28]. Researchers have proposed to include age structure of the harvested population, and earlier studies of optimal harvesting of agestructured fish populations have been published both in fishery ecology and economics. Leslie developed the theory for age-structured populations based on matrix methods [3]. Clark used bioeconomic modeling to integrate population dynamics with characterizations of important economic processes, and his book is the most comprehensive treatment of dynamic renewable resource models [4]. Clark [4] first studies fishing for a single age class, or cohort, with the goal to find the optimal time to harvest the entire cohort. Then he formulates a multicohort model with fixed recruitment and non-selective fishing mortality, the goal is to maximize present value profit, he finds the solutions to be a sequence of impulse controls. Wilen [38, 39] also pointed out more realistic fishery models should be based on population age structure. For many fish species, the von Bertalanffy formula [36] provides a good relation between age and size, and thus age-structured models are also size-structured.

\footnotetext{
${ }^{1}$ Department of Mathematical Sciences and Center for Computational Science, Middle Tennessee State University, Murfreesboro, TN

${ }^{2}$ Department of Mathematics, University of Tennessee and National Institute for Mathematical and Biological Synthesis, Knoxville, TN

${ }^{3}$ Fachbereich Mathematik/Informatik, Universität Osnabrück, Osnabrück, Germany

${ }^{*}$ Correspondence: wandi.ding@mtsu.edu
} 
Reed [24] studies optimal age-specific harvesting policy for maximizing equilibrium yield. He finds the optimal policy is of the "two-age" type. Getz has a series of papers on optimal harvesting with age structure [7], he and Haight [8] gave an extensive survey about agestructured population models and harvesting. Most studies they cite solve the problems under certain restrictions, such as requiring the harvest is constant over time. In Horwood and Whittle [11, 12], the optimal solutions are approximated by linearizing the optimality conditions in the neighborhood of the steady state with the goal to maximize a discounted yield for a multicohort fishery harvesting problem.

Stage [31] and Tahvonen [32, 34, 35, 33] suggest that including population age structure into economic studies deserves more emphasis. Stage [31] applies an age-structured model for Namibian linefishing and finds that the main result depends on the length of the planning horizon. Recently Tahvonen [32, 34, 35, 33] has analyzed models of optimal harvesting fish stocks with age structure. He studies the effects of different types of gear selectivity. One type is non-selective fishing gear, which means that all age classes are harvested in fixed but not necessarily equal proportions, and the optimal harvesting strategy may be "pulse-fishing" in time. The other type is "knife-edge" selectivity, which means all age classes above a certain age are subject to fishing mortality, while all younger fish completely escape. Tahvonen [35] also points out population age structure contains information on future harvest possibilities, where a biomass (or surplus production) model neglects this information and may lead to major deviations between the expected and actual outcomes especially under multiple steady states and nonlinearities.

$\mathrm{Li}$ and Yakubu [15] present a juvenile-adult discrete time production model of exploited fishery systems with general recruitment functions, and they use historical fish population data from Georges Bank to investigate the impact of recent harvesting levels on the sustainability of cod fishery. They show that a constant harvesting policy with the same harvesting rate of 2007 would lead to the recovery and sustainability of Georges Bank cod fishery. Skonhoft et al. [30] studies an age structured fishery model with two fishing fleets targeting two different mature age classes of the fish stock, an age class of immature fish is also included. The optimal harvesting policy essentially depends on the various biological (recruitment and survival) and economic (cost and price) parameters of the fishery. Their results differ significantly from a biomass model. They point out even when an age structured model is formulated in its simplest form and studied within an equilibrium context, no clear-cut results can be obtained even concerning the qualitative structure of the optimal harvesting, e.g., whether harvesting all or only some of year classes.

Though a complete model should take into account many species, other external biological factors and economic features, we will concentration on the development of a fisheries model for a single species, like cod, herring or plaice. Our model will be nonlinear due to density dependent effects only in the juvenile class since density effects have not been found for adults. In our discrete time model with age-structure, we are looking for optimal harvesting strategies, including the level of effort and the size of the nets. Optimal control of discrete populations has been used much less frequently than in systems of ordinary differential equations, and we use the tool of optimal control theory for discrete systems [14, 22, 29] to solve optimal harvesting problems for our model.

This paper formulates an age-structured fishery population model with nonlinear recruitment. We have $k$ age classes, the catching of fish with age greater than or equal to $m$ corresponds to an implicit net size (more precisely, the size of the fishing nets). The net parameter $m$ marks the width of the meshes of the fishing nets which catch a fish of age more than or equal to $m$. Again, we remark that age can easily be related to size [36]. With net-fishing, it is agewise nonselective, so the harvesting strategy will be the same for all age classes greater than or equal to $m$. Our goal is to maximize the profit (total gain) of fishing, and we assume the cost is directly proportional to the fishing effort. Our optimal control problem consists of finding both the optimal net size and the corresponding optimal harvesting strategies for each age class. We view our work going beyond the work of $[24,7,8,6]$ and concentrating on non-equilibrium dynamics. 
In Section 2, we present the age-structured fishery harvesting model. In Section 3 we derive the adjoint equations and the characterization of the optimal harvesting strategy. The model is applied to the case of a cod population in numerical simulations for Beverton-Holt and Ricker recruitment functions in Section 4. Our novel feature is to find both the optimal net size $m^{*}$ and the optimal fishing effort for each age class. Finally we draw conclusions in Section 5 .

\section{Age-structured Fishery Harvesting Model}

\subsection{Population Model}

Here we consider a one species fishery population model that takes the age structure and net harvesting into account. We will find the optimal harvesting strategy for a marine fish with $k$ age classes: larvae, juvenile 1 , juvenile $2, \ldots$, and adult fish.

We consider marine fish (like cod, herring, plaice, haddock or anchovy) that follow a yearly cycle, in which the adult fish migrate from the open sea to the spawning grounds. These spawning grounds are usually better protected areas in shallower waters closer to the coast. Most of the year the adult fish are in the open sea, which offers more food. They lay a very large number of eggs (for example, cod lay several million). From these the fish larvae hatch. Within several months these larvae metamorphose into juvenile fish. The larvae as well as the young fish live on plankton. Their size and demands for growth require large amount of food. Therefore the life cycle of fish larvae is intimately tied to the plankton bloom. Many of the larvae die of starvation or are eaten. The juvenile fish will stay in more protected areas (sometimes called nursery grounds) until they join the class of mature fish in their migrations. Thus the density dependence in the population dynamics will act essentially only during the larvae and possibly early juvenile stage.

The most important and most critical role in fish population dynamics is played by the recruitment function. Recruitment describes the development process from eggs to the first year class. Despite its importance, the recruitment process is as yet poorly understood. The larval stage makes the recruitment a nonlinear process [5]. If $R$ stands for this relationship, the following properties for $R$ are natural:

1. linear increase at low larval concentration,

2. saturation at high larval concentration.

In this paper, we will use for illustration the most common forms: Beverton-Holt and Ricker. However, the dynamics resulting from these two forms may be quite distinct. To decide which is better suited would depend on population density data. In our analysis, we will take a general form for $R$, later in numerical simulations, we will use those two forms for recruitment, representing two different populations.

The yearly cycle of marine fish suggests to describe their dynamics as a discrete age class model. We assume that we have the age classes $1,2, \ldots, k$ of length $T_{1}, T_{2}, \ldots, T_{k}$, with $T_{i} \in \mathbb{N}$. Let $x_{i}(t)$ denote the number of fish in age class $i$ at time $t$. Then our model is

$$
\begin{aligned}
x_{1}(t+1) & =\left(1-\mu_{1}\right)\left(1-\frac{1}{T_{1}}\right) x_{1}(t)+R\left(x_{l}(t), \ldots, x_{k}(t)\right), \\
x_{i}(t+1) & =\left(1-\mu_{i-1}\right) \frac{1}{T_{i-1}} x_{i-1}(t)+\left(1-\mu_{i}\right)\left(1-\frac{1}{T_{i}}\right) x_{i}(t) \quad \text { for } i=2, \ldots, k-1, \\
x_{k}(t+1) & =\left(1-\mu_{k-1}\right) \frac{1}{T_{k-1}} x_{k-1}(t)+\left(1-\mu_{k}\right) x_{k}(t) .
\end{aligned}
$$

Here $\mu_{i}$ is the mortality in class $i$. The term $\left(1-\mu_{i}\right)\left(1-\frac{1}{T_{i}}\right)$ is the fraction of individuals in age class $i$ who survive and remain in class $i$ after one time unit, and $\left(1-\mu_{i-1}\right) \frac{1}{T_{i-1}}$ is the fraction that survives and moves to the next age category $i$. The nonlinear recruitment function $R\left(x_{l}(t), \ldots, x_{k}(t)\right)$ depends on $x_{l}(t), \ldots, x_{k}(t)$ in general. Here we assume the fertility begins with class $l$. 
Since the number of eggs of an adult female fish is proportional to its weight, the number of eggs in the year $t$ is given by

$$
E(t)=c \sum_{i=l}^{k} x_{i}(t) w_{i}(t),
$$

where $c$ is a proportionality constant, i.e., the number of viable eggs per weight and $w_{i}(t)$ denotes the average weight of a fish in age class $i$ at time $t$. The number of recruits becomes

$$
R\left(x_{l}(t), \ldots, x_{k}(t)\right)=\frac{a E(t)}{1+\frac{E(t)}{E_{0}}}
$$

for Beverton-Holt recruitment and

$$
R\left(x_{l}(t), \ldots, x_{k}(t)\right)=a E(t) e^{-\frac{E(t)}{E_{0}}}
$$

for Ricker recruitment, where $a, E_{0}>0$ are constants. Note that the only nonlinearity of this model is in the stock-recruitment function $R\left(x_{l}(t), \ldots, x_{k}(t)\right)$.

The system in (2.1) describes a dynamical system, which has been studied in itself in order to understand the basic parameters and its stability, see [16] for details. One finds that for realistic values of the parameter $a$, there is a unique globally stable equilibrium for both recruitment functions.

\subsection{Optimal Harvesting Model}

Let $u(t)$ be the fishing effort, which would depend on the number of fish fleets and equipment. Let $0 \leq u \leq M$, with $M$ the maximum fishing effort. We are assuming that "on average" the size is an increasing function of the age. The catching of fish of age greater than or equal to $m$ corresponds to an implicit net size. The net parameter $m$ marks the width of the meshes of the fishing nets which catch a fish of age more than or equal to $m$. Then the harvesting of each age class is

$$
h_{i}(t)= \begin{cases}0, & i=1,2, \ldots, m-1 \\ u(t), & i=m, m+1, \ldots, k\end{cases}
$$

This desribes a type of impulse fishing at the end of year $t$. Our control set is

$$
U=\{u=(u(1), \ldots, u(T-1)) \mid 0 \leq u(t) \leq M, t=1, \ldots, T-1\},
$$

where $M \leq 1$ and $T$ is the total number of years of fishing. We have the following discrete harvesting model with the general nonlinear recruitment $R\left(x_{l}(t), \ldots, x_{k}(t)\right)$ :

$$
\begin{aligned}
x_{1}(t+1) & =\left(1-\mu_{1}\right)\left(1-\frac{1}{T_{1}}\right) x_{1}(t)+R\left(x_{l}(t), \ldots, x_{k}(t)\right)-h_{1}(t) x_{1}(t), \\
x_{i}(t+1) & =\left(1-\mu_{i-1}\right) \frac{1}{T_{i-1}} x_{i-1}(t)+\left(1-\mu_{i}\right)\left(1-\frac{1}{T_{i}}\right) x_{i}(t)-h_{i}(t) x_{i}(t), \\
x_{k}(t+1) & =\left(1-\mu_{k-1}\right) \frac{1}{T_{k-1}} x_{k-1}(t)+\left(1-\mu_{k}\right) x_{k}(t)-h_{k}(t) x_{k}(t) .
\end{aligned}
$$

for $i=2, \ldots, k-1$, and $h_{i}(t), i=1, \ldots, k$ is from $(2.5)$. We could have different $u_{i}(t)$ for each age class, but in reality, using net-fishing, the harvesting is agewise nonselective. We choose the mesh $m$ size to be an integer, so that we do not have a partial catch in any class.

We will maximize the total gain from fishing for $t=1,2, \ldots, T-1$ while maximizing the fish population at the final time $T$ for $i=m, \ldots, k$ :

$$
G(m, u)=\sum_{i=m}^{k} A x_{i}(T)+\sum_{t=1}^{T-1}\left(\sum_{i=m}^{k} u(t) x_{i}(t) w_{i} p_{i}-B u(t)\right), u \in U .
$$


If one is also trying to maintain a reasonable level of fish in the harvested classes (to benefit the fish population), then the coefficient $A$ is positve; otherwise if concentrating only on gain, then $A$ would be 0 . For $p_{i}$, the price per unit weight of fish from class $i$ at time $t$, we will use

$$
p_{i}=p_{0} w_{i}^{\alpha},
$$

with $\alpha>1, p_{0}>0$, since larger fish give more fillets and they are more valuable. We assume the cost is directly proportional to the fishing effort, and the size of coefficient $A$ relative to the $\operatorname{cost} B$ of the fishing effort balances the relative importance of maximizing the final fish population and the cost of the fishing effort. Notice the objective functional has linear dependence of the control $u(t)$, which will lead to a bang-bang and/or singular optimal control problem, and we only harvest those who pass the net with size $m$.

Then the optimal harvesting solution algorithm consists of two parts: first, for each $m$ fixed, we seek to find the corresponding optimal harvesting strategy, i.e., the optimal fishing effort $u$ to maximize $G(m, u)$; second, we find the optimal net mesh size $m$ which gives the largest total gain. Since we only have a finite number of age classes, we can compare the $G(m, u)$ values for different $m$ in order to obtain the optimal net size. Our novel feature is to find both the optimal net size and the optimal fishing effort.

We will use the extension of Pontryagin's Maximum Principle (PMP) [14, 22, 29] for the optimal control of discrete system. The technique involves the use of adjoint functions, which append the discrete system (2.6) to the maximization of the objective functional (2.7). PMP gives the optimality system of difference equations consisting of the state and adjoint difference equations coupled with an optimal control characterization. Note that the adjoint equations have transversality conditions at the final time while the state equations have initial conditions. The key idea is that the adjoint method provides us with the gradient of the cost function needed for the maximization procedure. We note that an optimal control exists due to the finite dimensional structure of this system.

\section{$3 \quad$ Adjoint Equations}

Note that we are temporarily assuming that the parameter $m$ is fixed and later we will compare the $G(m, u)$ values to find the optimal net size $m$. Since there are a finite number of values for $m$, we will run our numerical results for all values of $m$ and see which gives the largest gain.

To simplify our notation, we denote $\sigma_{i}=\left(1-\mu_{i}\right)\left(1-\frac{1}{T_{i}}\right), i=1, \ldots, k-1$ and

$$
\tau_{i}=\left(1-\mu_{i-1}\right) \frac{1}{T_{i-1}} \quad \text { for } i=1, \ldots, k-1 .
$$

Theorem 3.1. Given $m \geq l$, and an optimal control $u^{*}$ and the corresponding solutions $x_{i}, i=1, \ldots, k$ of the state system (2.6), there exist adjoint variables $\lambda_{i}, i=1, \ldots, k$ satisfying: for $t=1, \ldots, T-1$,

for $1 \leq j \leq l-1$,

$$
\lambda_{j}(t)=\lambda_{j}(t+1) \sigma_{j}+\lambda_{j+1}(t+1) \tau_{j}
$$

for $l \leq j \leq m-1$,

$$
\lambda_{j}(t)=\lambda_{j}(t+1) \sigma_{j}+\lambda_{j+1}(t+1) \tau_{j}+\lambda_{1}(t+1) \frac{\partial R(t)}{\partial x_{j}} ;
$$

for $m \leq j \leq k-1$,

$$
\begin{aligned}
\lambda_{j}(t)=\lambda_{j} & (t+1) \sigma_{j}+\lambda_{j+1}(t+1) \tau_{j}+\lambda_{1}(t+1) \frac{\partial R(t)}{\partial x_{j}} \\
& -\lambda_{j}(t+1) u(t)+u(t) w_{j} p_{j}
\end{aligned}
$$


for $j=k$,

$$
\begin{gathered}
\lambda_{k}(t)=\lambda_{k}(t+1)\left(1-\mu_{k}\right)+\lambda_{1}(t+1) \frac{\partial R(t)}{\partial x_{k}} \\
-\lambda_{k}(t+1) u(t)+u(t) w_{k} p_{k}
\end{gathered}
$$

with $\lambda_{j}(T)=0, j=1, \ldots, m-1$, and $\lambda_{j}(T)=A, j=m, \ldots, k$.

Proof. The Hamiltonian for $t=1,2, \ldots, T-1$ is

$$
\begin{aligned}
H(t)= & \left(\sum_{i=m}^{k} u(t) x_{i}(t) w_{i} p_{i}-B u(t)\right) \\
& +\lambda_{1}(t+1)\left[\sigma_{1} x_{1}(t)+R\left(x_{l}(t), \ldots, x_{k}(t)\right)-h_{1}(t) x_{1}(t)\right] \\
& +\sum_{i=2}^{k-1} \lambda_{i}(t+1)\left[\tau_{i-1} x_{i-1}(t)+\sigma_{i} x_{i}(t)-h_{i}(t) x_{i}(t)\right] \\
& +\lambda_{k}(t+1)\left[\tau_{k-1} x_{k-1}(t)+\left(1-\mu_{k}\right) x_{k}(t)-h_{k}(t) x_{k}(t)\right] .
\end{aligned}
$$

Rewrite $H(t)$ as

$$
\begin{aligned}
H(t)=( & \left.\sum_{i=m}^{k} u(t) x_{i}(t) w_{i} p_{i}-B u(t)\right)+\sum_{i=1}^{k-1} \lambda_{i}(t+1) \sigma_{i} x_{i}(t) \\
& +\sum_{i=1}^{k-1} \lambda_{i+1}(t+1) \tau_{i} x_{i}(t)-\sum_{i=m}^{k} \lambda_{i}(t+1) u(t) x_{i}(t) \\
& +\lambda_{1}(t+1) R\left(x_{l}(t), \ldots, x_{k}(t)\right)+\lambda_{k}(t+1)\left(1-\mu_{k}\right) x_{k}(t) .
\end{aligned}
$$

Using the extension of Pontryagin's Maximum Principle [14, 22, 29], the necessary conditions are for $j=1,2, \ldots, k, t=1,2, \ldots, T-1, m \geq l$,

$$
\begin{aligned}
\lambda_{j}(t) & =\frac{\partial H(t)}{\partial x_{j}} \\
\lambda_{j}(T) & = \begin{cases}0, & j=1, \ldots, m-1 ; \\
A, & j=m, \ldots, k ;\end{cases}
\end{aligned}
$$

For $1 \leq j \leq l-1$,

$$
\lambda_{j}(t)=\lambda_{j}(t+1) \sigma_{j}+\lambda_{j+1}(t+1) \tau_{j}
$$

for $l \leq j \leq m-1$,

$$
\lambda_{j}(t)=\lambda_{j}(t+1) \sigma_{j}+\lambda_{j+1}(t+1) \tau_{j}+\lambda_{1}(t+1) \frac{\partial R(t)}{\partial x_{j}} ;
$$

for $m \leq j \leq k-1$,

$$
\begin{aligned}
\lambda_{j}(t)=\lambda_{j} & (t+1) \sigma_{j}+\lambda_{j+1}(t+1) \tau_{j}+\lambda_{1}(t+1) \frac{\partial R(t)}{\partial x_{j}} \\
& -\lambda_{j}(t+1) u(t)+u(t) w_{j} p_{j}
\end{aligned}
$$

for $j=k$,

$$
\begin{gathered}
\lambda_{k}(t)=\lambda_{k}(t+1)\left(1-\mu_{k}\right)+\lambda_{1}(t+1) \frac{\partial R(t)}{\partial x_{k}} \\
-\lambda_{k}(t+1) u(t)+u(t) w_{k} p_{k}
\end{gathered}
$$


When we specify a recruitment function in the numerical results section, we will use maximizing the Hamiltonian with respect to the control and the sign of the switching function,

$$
\frac{\partial H(t)}{\partial u(t)} \text { at } u^{*}
$$

to determine the optimal control.

Note that we only have a finite number of age classes, we can compare the $G(m, u)$ values for different $m$ in order to obtain the optimal net size.

\section{Numerical Results}

The characterization of the optimal control $u^{*}$ depends on the sign of this switching function:

$$
\begin{gathered}
S=\sum_{i=m}^{k}\left(p_{i} w_{i}\left(-\lambda_{i}(t+1)\right) x_{i}(t)-B,\right. \\
u^{*}(t)=0, \text { if } S(t)<0 \text { at } t ; \\
u^{*}(t)=M, \text { if } S(t)>0 \text { at } t .
\end{gathered}
$$

For the singular case, when $S(t)=0$, we calculate the optimal control value at that $t$ numerically by maximizing the Hamiltionian at that time.

To solve the optimal harvesting problem numerically, due to the boundary conditions being at the initial time for the states and at the final time for adjoints, an iterative method is used to solve this optimality system. Given initial guesses for the controls and the state equations, the state system (2.6) is solved forward in time, and the adjoint system (3.1)(3.4) is solved backward in time. The control is updated using the characterization (4.2) with the newly found state and adjoint values, and the iteration repeats until convergence occurs. See the book by Lenhart and Workman [14] for details of this method.

Note the linear dependence of the control $u$ in the objective functional (2.7) will give a bang-bang and/or singular case of the optimal control. We would like to point out that we will find the optimal net size based on the maximum total gain from fishing among the finite number of $m$ values and the corresponding optimal fishing effort for each age class. We note that using $m$ as a function of time is not reasonable since changing the net mesh sizes each year is unrealistic.

To apply this to a specific population in a certain location, one should know which recruitment function is a better fit for that population. We will use Beverton-Holt and Ricker's recruitment functions in our simulations. In (3.2)-(3.4), for $l \leq j \leq k$,

$$
\frac{\partial R(t)}{\partial x_{j}}= \begin{cases}\frac{a c w_{j}}{\left[1+\frac{E(t)}{E_{0}}\right]^{2}} & \text { Beverton-Holt form, } \\ a c w_{j} \cdot e^{-\frac{E(t)}{E_{0}}}\left(1-\frac{E(t)}{E_{0}}\right) & \text { Ricker form. }\end{cases}
$$

As noted above, we will consider cod in these simluatons and optimal control calculations. Cod is thus the typical prototype marine fish, other species can be analyzed along the same lines. For parameter values besides the recruitment functions, see Myers [17, 1, 19, 18, 20]. The maximum age for cod is 30 years and fertility begins at about $t=8$ to 12 years. So we choose 12 age classes, for the first age class, $T_{1}=8$ and for other classes, $T_{i}=2, i=2, \ldots 12$. The Bertalanffy weight formula [36] as a function of class $i$ is

$$
w_{i}=17\left(1-e^{-0.12(2 i+6)}\right)^{3} .
$$

The average mortality for cod is about $\mu=0.18$, and we used a linear function to make $\mu$ increase between 0.18 and 0.22 as the age classes increase, to account for increasing mortality. 
Also recall the price per unit weight of fish is

$$
p_{i}=p_{0} w_{i}^{\alpha},
$$

with $\alpha>1, p_{0}>0$.

We assumed catching the fish of age greater than or equal to $m$ corresponds to an implicit net size parameter. We set $l=2$ to indicate fertility begins at age class 2 and we set $m \geq l$. After we find the optimal harvesting strategy for each fixed $m$, we vary $m=2, \ldots, 12$ to find the optimal net size in order to obtain the largest $G(m, u)$ value. We set the lower bound for harvesting to be 0 and the upper bound $M=0.5$. The parameters in the nonlinear recruitment functions are from Li and Yakubu [15] using Northeast Fisheries Science Center data for the assessment of 19 Northeast Ground-fish Stocks through 2007 [21]. For BevertonHolt recruitment, $a=1.13, \frac{1}{E_{0}}=1.012 \times 10^{-5}, c=10^{5}$, and for Ricker recruitment $a=1.851 \times 10^{-4}, \frac{1}{E_{0}}=6.262 \times 10^{-5}, c=10^{6}$. Note that these two recruitment functions represent different sets of data from different populations, give quite different dynamics and should not be directly compared. We use initial conditions of $10^{5}$ for class 1 and $10^{3}$ for the other classes for the Beverholt-Holt case and $10^{6}$ for class 1 and $10^{4}$ for the other classes for the Ricker case. The population with Beverton-Holt recruitment has a much higher level near the end of the 30 years.

As in [30], we examine how the optimal harvesting policy depends on the biological (recruitment) and economic (cost and price) parameters of the fishery. First we give numerical results for Beverton-Holt recruitment function. For $B=10^{4}, A=10^{4}, \alpha=1.1, T=30$, the optimal net size is $m=2$. When $\alpha=1.5$ and 2 the corresponding $m$ becomes 3 and 4 , respectively. For $A=10^{4}$, the optimal control is approximately bang-bang; we do not harvest the whole time interval due to some emphasis on saving the fish level at the final time. As $\alpha$ increases, the price goes up (especially for older fish), the optimal net size $m$ increases, and we spend longer time to harvest with the maximum effort. Figure (1) gives the optimal harvesting strategies for three different scenarios, and Figure (3) shows the cod population corresponding to the case $1(\mathrm{a})$.

For $A=0$, i.e., we only want to maximize the profit, when $T=30$, the optimal net size $m=2$, and the optimal strategy is to harvest with the maximum fishing effort for the entire fishing period, see Figure 2(a). This maximum effort makes sense in this scenario without consideration of the unharvested fish at the final time. When $T=20$, Figure 2(b) gives the optimal harvesting strategy and optimal net size for $B=10^{5}, A=0, \alpha=4$. In this case $m=4$ and we see the bang-bang optimal control with 2 switches. Switching twice may be due to the playoff between the value of large fish due to increased $\alpha$ (from 1.1 to 4 ) and the increased cost $B$ (from $10^{4}$ to $10^{5}$ ). Maybe the period of no harvest gives the population some time to grow larger, more valuable fish. We also examined a variety of different parameter values, when $B$ increases, we spend shorter time harvesting at the maximum level since it is more expensive. When $A$ decreases, i.e., maximizing the fish population at the final time is less important, we spend longer time harvesting at the maximum level in order to maximize the profit.

Next, we give numerical simulations for Ricker recruitment. For $T=30, B=10^{5}$, $A=10^{4}$, we vary $\alpha=1.1,1.5$, and 2.5 the corresponding optimal net size $m=3,3$ and 4 , respectively. The optimal harvesting pattern is similar to Beverton-Holt case; see Figure (4). When $B=10^{4}, A=0, \alpha=2.5$, then $m=4$ and the unusual switching pattern in the Figure 4(d) may again illustrate the playoff between price $\alpha$ and cost $B$. In Figure (3) with Beverton-Holt recruitment, the population levels off, while in Figure (5) the population decreases quickly. Different dynamics are expected due to these 2 different recruitment functions. Note Figure (5) corresponds to the case 4(a). These low population levels indicate that an upper bound of 0.5 on the harvest of a fish stock with Ricker recruitment is too large; when an upper bound of 0.1 is used, the population levels in some ages classes increase by 1000 . 


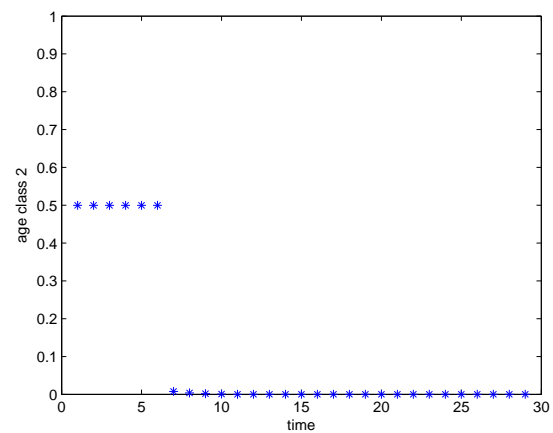

(a) Optimal Harvest, $m=2, \alpha=1.1$

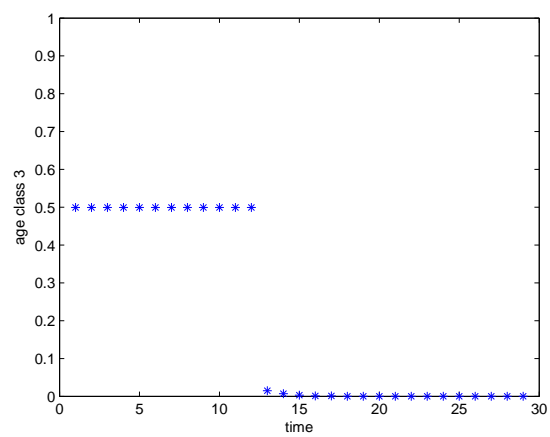

(b) Optimal Harvest, $m=3, \alpha=1.5$

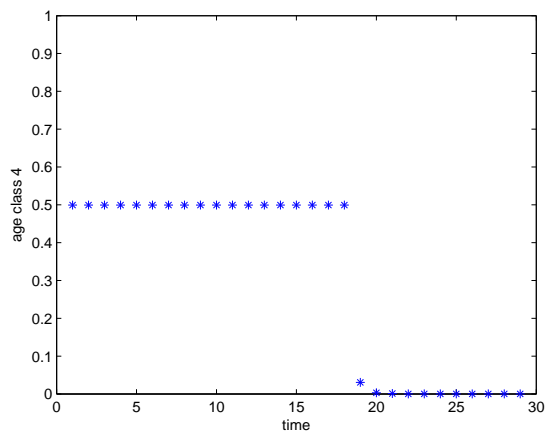

(c) Optimal Harvest, $m=4, \alpha=2$

Figure 1: Optimal harvest with Beverton-Holt recruitment for $T=30, B=10^{4}, A=10^{4}$ with different $\alpha$, (a) optimal net size $m=2$ when $\alpha=1.1$, (b) $m=3$ when $\alpha=1.5$, and (c) $m=4$ when $\alpha=2$.

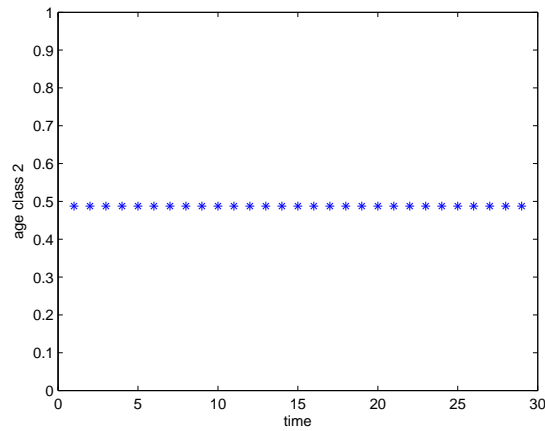

(a) Op. Harvest, $m=2, T=30, \alpha=1.1, B=10^{4}$

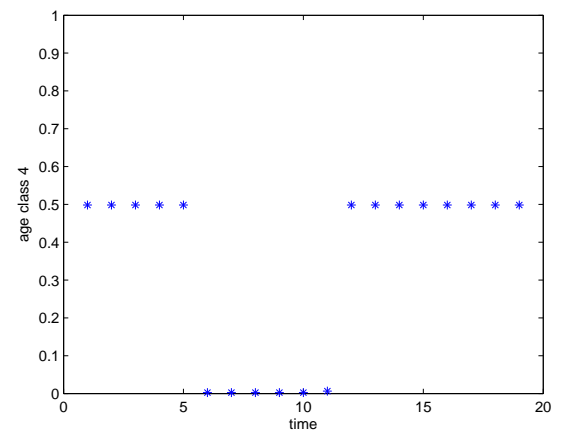

(b) Op. Harvest, $m=4, T=20, \alpha=4, B=10^{5}$

Figure 2: Comparing optimal harvest with Beverton-Holt recruitment. 

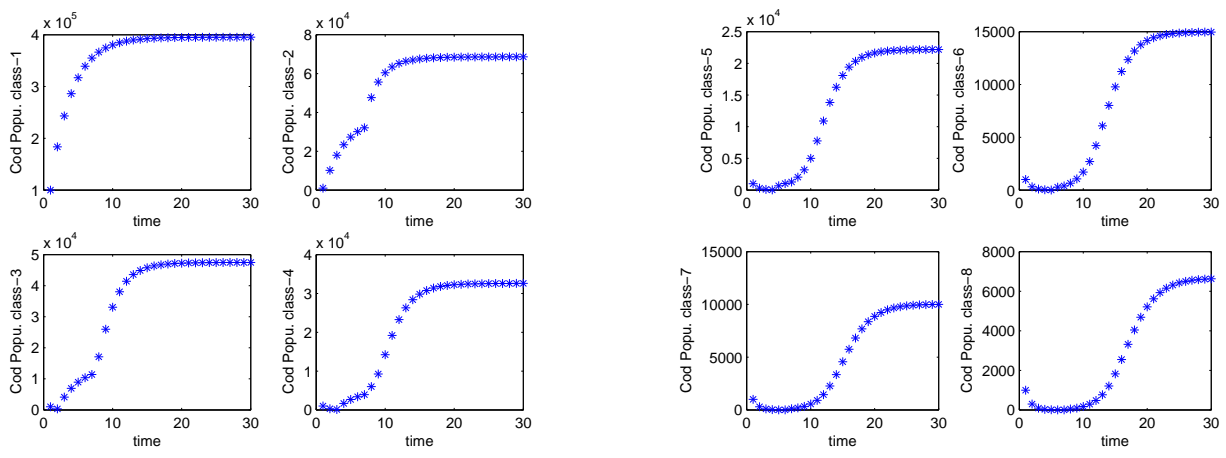

(a) Cod Population for age classes 1-4
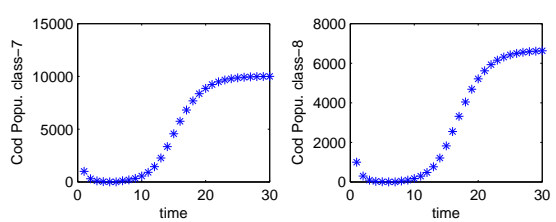

(b) Cod Population for age classes 5-8
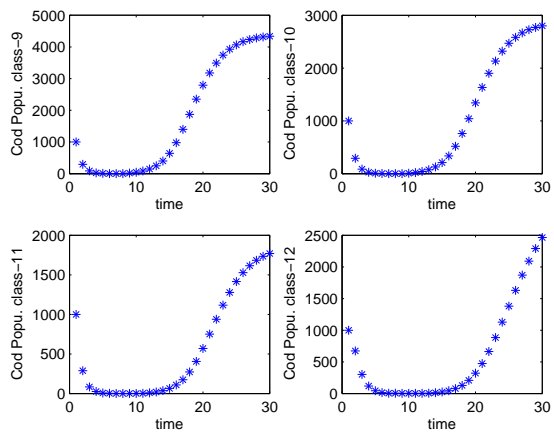

(c) Cod Population for age classes 9-12

Figure 3: Cod population with Beverton-Holt Recruitment for age classes 1-12 after harvesting, $B=10^{4}, A=10^{4}, \alpha=1.1, T=30$, corresponding to Figure 1(a). 


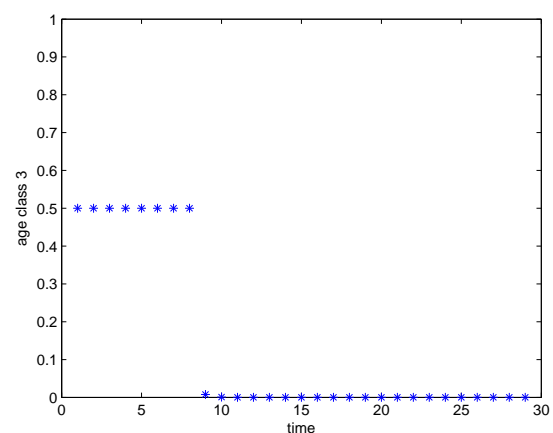

(a) Optimal Harvest, $m=3, \alpha=1.1, A=10^{4}$

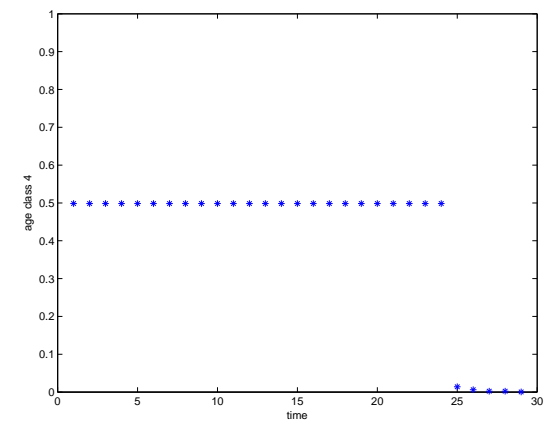

(c) Optimal Harvest, $m=4, \alpha=2.5, A=10^{4}$

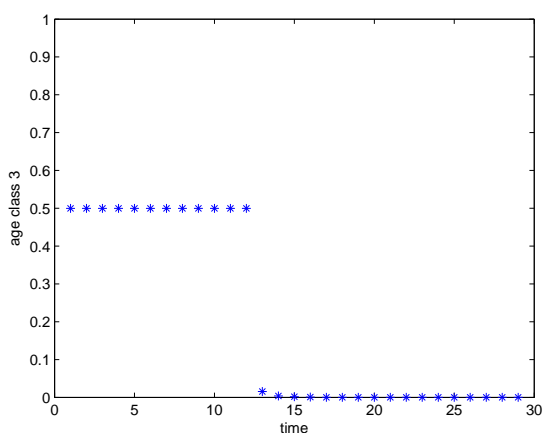

(b) Optimal Harvest, $m=3, \alpha=1.5, A=10^{4}$

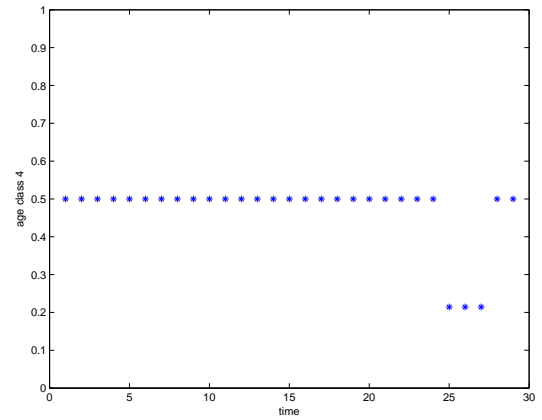

(d) Optimal Harvest, $m=4, \alpha=2.5, A=0$

Figure 4: Optimal harvesting with Ricker recruitment for $T=30$, (a) optimal net size $m=3$ when $\alpha=1.1, B=10^{5}, A=10^{4}$, (b) $m=3$ when $\alpha=1.5, B=10^{5}, A=10^{4}$, (c) $m=4$ when $\alpha=2.5, B=10^{5}, A=10^{4}$, and (d) $m=4$ when $\alpha=2.5, B=10^{5}, A=0$. 

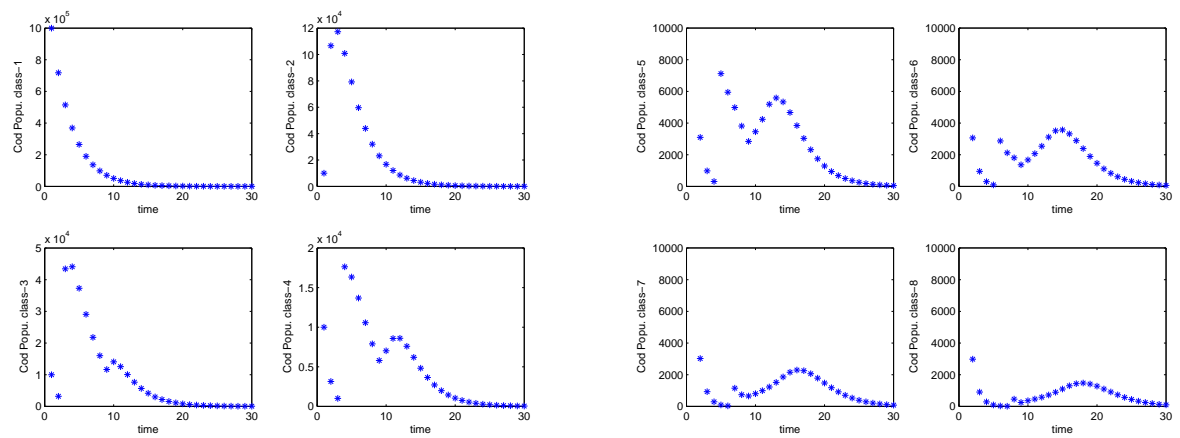

(a) Cod Population for age classes 1-4

(b) Cod Population for age classes 5-8
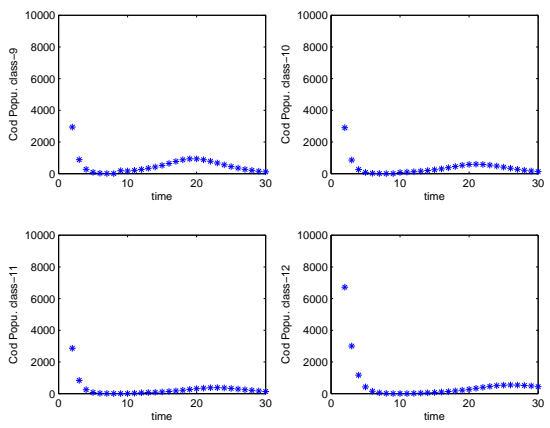

(c) Cod Population for age classes 9-12

Figure 5: Cod population with Ricker Recruitment for age classes 1-12 after harvesting, $B=10^{5}, A=10^{4}, \alpha=1.1, T=30$, corresponding to Figure 4(a) 


\section{Conclusions}

We applied optimal control theory for an age structured discrete harvest model with nonlinear recruitment. The feature of our project is to find both the optimal net size and the corresponding optimal harvesting strategies for each age class. We derived the adjoint equations and the characterization of the optimal control using the extension of Pontryagin's Maximum Principle [22] for the general nonlinear recruitment, then we've implemented numerical simulations for Beverton-Holt and Ricker recruitment functions. The parameters in the nonlinear recruitment functions are from $\mathrm{Li}$ and Yakubu [15] using Northeast Fisheries Science Center data for the assessment of 19 Northeast Ground-fish Stocks through 2007 [21]. As in [30], we've examined how the optimal harvesting policy depends on the biological (recruitment) and economic (cost and price) parameters of the fishery. If the goal includes maximizing the fish population at the final time, then a bang-bang optimal control occurs; if we only want to maximize the profit, then the optimal harvesting strategy is to harvest with the maximum effort for the entire fishing period unless the $\operatorname{cost} B$ is very high. We notice the optimal net size can be affected by the price parameter $\alpha$. The optimal harevest effort may be affected by a playoff between costs and the price parameter $\alpha$, and thus an intermediate time interval without any harvesting could occur.

\section{Acknowledgements}

Ding's work was supported by Faculty Research and Creative Activities (FRCAC) at Middle Tennessee State University.

Lenhart's work was partially supported by the National Institute for Mathematical and Biological Synthesis, sponsored by the National Science Foundation, the U. S. Department of Homeland Security, and the U.S. Department of Agriculture through NSF Award EF-0832858, with additional support from the University of Tennessee, Knoxville. Lenhart is also partially supported by the UT Center for Business and Economic Research.

\section{References}

[1] N. J. Barrowman and R. A. Myers, 2000. Still more spawner-recruitment curves: the hockey stick and its generalizations. Can. J. Fish. Aquat. Sci. 57: 665-676.

[2] R. J. Beverton and S. J. Holt, 1957. On the Dynamics of Exploited Fish Populations, Fisheries Investigation Series II, Vol. 19, Ministry of Agriculture, Fisheries and Food, London.

[3] H. Caswell, 2000. Matrix Population Models, Construction, Analysis and Interpretation. Sinauer Associates, Inc. Publishers, Sunderland, MA.

[4] C. Clark, 2005. Mathematical Bioeconomics: The Optimal Management of Renewable Resources, 2nd edition, Wiley.

[5] D. H. Cushing, 1981. Fisheries Biology, A Study in Population Dynamics, Univ. of Wiscon. Press.

[6] B. Dacorogna and F. Weissbaum, 2002. Maximum sustainable yield with continuous age structure and density-dependent recruitment, Math. Biosciences, 120:1:99-126.

[7] W. Getz, 1985. Optimal and feedback strategies for managing multicohort populationes. J. Optim. Theory Appl. 46:505-514.

[8] W. M. Getz and R. G. Haight, 1989. Population Harvesting: Demographic Models for Fish, Forest and Animal Resources, Princeton, NJ: Princeton University Press. 
[9] R. Hilborn, 2012. Overfishing: What Everyone Needs to Know. Oxford University Press, Oxford.

[10] R. Hilborn and C. J. Walters, 1992. Quantitative Fisheries Stock Assessment: Choice, Dynamics and Uncertainty. Chapman \& Hall, Inc., London.

[11] J. W. Horwood and P. Whittle, 1986. Optimal Control in the Neighbourhood of an Optimal Equilibrium with Examples from Fisheries Models. IMA J. Math. Appl. Med. Bio. 3:129-142.

[12] J. W. Horwood and P. Whittle, 1986. The Optimal Harvest from a Multicohort Stock. IMA J. Math. Appl. Med. Bio. 3:143-155.

[13] M. Kurlansky, 1998. Cod: A biography of the fish that changed the world. Penguin Books, London.

[14] S. Lenhart and J. Workman, 2007. Optimal Control Applied to Biological Models, Chapman Hall/CRC, Boca Raton.

[15] N. Li and A-A Yakubu, 2012. A Juvenile-Adult Discrete-Time Production Model of Exploited Fishery Systems, Natural Resource Modeling, 25:2, 273-324.

[16] M. K. S. Mohamed, 2005. Harvesting of Age Structured Fish Populations, Dissertation, University of Osnabrück, Germany.

[17] R. A. Myers, 2001. Stock and recruitment: generalizations about maximum reproductive rate, density dependence, and variability using meta-analytic approaches. ICES Journal of Marine Science, 58:937-951.

[18] R. A. Myers, N. J. Barrowman, J. A. Hutchings, and A. A. Rosenberg, 1995. Population Dynamics of Exploited Fish Stocks at Low Population Levels. Science, vol. 269:11061108.

[19] R. A. Myers, K. G. Bowen, and N. J. Barrowman, 1999. Maximum reproductive rate of fish at low population sizes. Can. J. Fish. Aquat. Sci. 56: 2404-2419.

[20] R. A. Myers and G. Mertz, 1997. Maximum population growth rates and recovery times for Atlantic Cod, Gadus morhua. Fishery Bulletin 95: 762-772.

[21] Northeast Fisheries Science Center, Assessment of 19 Northeast Ground-fish Stocks Through 2007, 2009. Report of the 3rd Groundfish Assessment Review Meeting (GARM III).

[22] L. S. Pontryagin, V. S. Boltyanskii, R. V. Gamkrelidze, and E. F. Mishchenko, 1962. The Mathematical Theory of Optimal Processes, Wiley-Interscience, New York.

[23] T. J. Quinn, 2003. Ruminations on the Development and Future of Population Dynamics Models in Fisheries, Natural Resource Modeling, 16:4, 341-392.

[24] W. J. Reed, 1980. Optimum Age-Specific Harvesting in a Nonlinear Population Model, BIOMETRICS 36, 579-593.

[25] W. E. Ricker, 1954. Stock and recruitment, J. Fish. Res. Board Can. 11:559-623.

[26] W.E. Ricker, 1958. Handbook of Computations for Biological Statistics of Fish Populations, Bull. 119, Fisheries Research Board Canada.

[27] M. B. Schaefer, 1954. Some aspects of the dynamics of populations important to the management of commercial marine fisheries, Inter-Am. Trop. Tuna Comm., Bull. 1:25-56. 
[28] M. B. Schaefer, 1957. Some considerations of population dynamics and economics in relation to the management of the commercial marine fisheries, J. Fish. Res. Board Can. 14:669-681.

[29] S. P. Sethi and G. L. Thompson, 2009. Optimal Control Theory: Applications to Management Science and Economics, 2nd edition, Springer.

[30] A. Skonhoft, N. Vestergaard, and M. Quaas, 2012. Optimal Harvest in an Age Structured Model with Different Fishing Selectivity, Environ Resource Econ, 51:525-544.

[31] J. Stage, 2006. Optimal Harvesting In an Age-Class Model with Age-Specific Mortalities: An Example from Namibian Linefishing, Natural Resource Modelling 19(4): 609-31.

[32] O. Tahvonen, 2008. Harvesting an Age-Structured Population as Biomass: Does it Work?, Natural Resource Modeling, 21:4:525-550.

[33] O. Tahvonen, 2009. Age-structured optimization models in fisheries bioeconomics: a survey, in: R. Boucekkine, N. Hritonenko, Y. Yatsenko (eds.), Optimal Control of Agestructured Populations in Economy, Demography, and the Environment, Routledge.

[34] O. Tahvonen, 2009. Economics of harvesting age-structured fish populations, Journal of Environmental Economics and Management, 58:281-299.

[35] O. Tahvonen, 2009. Optimal harvesting of age-structured fish populations, 24:147-169, Marine Resource Economics.

[36] L. von Bertalanffy, 1938. A quantitative theory of organic growth, Human Biology, 10:181-213.

[37] C. J. Walters and S. J. D. Martell, 2004. Fisheries ecology and management, Princeton, NJ, Princeton University Press.

[38] J. E. Wilen, 1985. Bioeconomics of Renewable Resource Use. Handbook of Natural Resource and Energy Economics, vo 1.1, A. V. Kneese and J. L. Sweeney, eds. Amsterdam: Elsevier.

[39] J.E. Wilen, 2000. Renewable Resource Economists and Policy: What Differences Have We Made? Journal of Environmental Economics and Management 39:306-27. 